\title{
Pulmonary arterial hypertension and right ventricular systolic dysfunction in COVID-19 survivors
}

\author{
Rosario Rossi ${ }^{1,2}$, Francesca Coppi ${ }^{1,2}$, Daniel Enrique Monopoli ${ }^{1}$, \\ Fabio Alfredo Sgura ${ }^{1}$, Salvatore Arrotti ${ }^{1}$, Giuseppe Boriani ${ }^{1}$ \\ ${ }^{1}$ Division of Cardiology, University of Modena and Reggio Emilia (UNIMORE), Modena, Italy \\ ${ }^{2}$ Pulmonary Hypertension Program, Azienda Ospedaliera Policlinico di Modena, Modena, Italy
}

Although much has been published about the in-hospital prognosis of COVID-19 patients, much less attention has been paid to what happens to those who have been discharged alive from the hospital [1]. In the present study we investigated a group of patients initially admitted for acute respiratory failure due to coronavirus disease 2019 (COVID-19)-related interstitial pneumonia and then, at a later time point, discharged from hospital. We noted that some of these patients presented a specific cluster of signs and symptoms: asthenia, fatigue, high heart rate (HR) at rest, and tachycardia disproportionate to physical exertion [2]. These patients find it difficult to resume their social and working life after discharge. We can say that these symptoms and signs fall within the generic definition of "post-COVID syndrome" [3], but we do not know the origin and causes of this clinical condition. We then performed a right heart catheterization (RHC) in all these patients, in order to evaluate the possible presence of cardiac and/or pulmonary vascular alterations.

The study group consisted of 25 consecutive patients, COVID-19 survivors, referred to our divisional outpatient clinic for the cluster of signs and symptoms described above. All patients were hospitalized between March 2020 and February 2021. In all these patients we performed a RHC and a 6 -minute walking test (6MWT). The obtained results were compared with those of a control group, which comprised 25 gender- and age-matched patients, COVID-19 survived, but asymptomatic.
Patients with pulmonary hypertension $(\mathrm{PH})$ underwent an additional 6MWT and catheterization every 6 months of follow-up.

The present study was approved by the local Ethical Committee (protocol number: AOU 0012597).

Right heart catheterization was performed using a $7 \mathrm{~F}$ balloon-tipped (Swan-Ganz catheter), triple lumen thermodilution catheters. Right atrial pressure (RAP), mean pulmonary artery pressure (mPAP), and pulmonary capillary wedge pressure (PCWP) were recorded. Cardiac output (CO) was determined by thermodilution method. Cardiac index $(\mathrm{CI}=\mathrm{CO} /$ body surface area $)$, right ventricle stroke volume $(\mathrm{RVSV}=\mathrm{CO} / \mathrm{HR})$, and pulmonary vascular resistance $[\mathrm{PVR}=(\mathrm{mPAP}-\mathrm{PCWP}) / \mathrm{CO}]$ were calculated. Right ventriculography was performed in the $30^{\circ}$ right anterior oblique view using a $6 \mathrm{~F}$ pig-tail catheter. Right ventricular (RV) end-diastolic diameter (EDD) and ejection fraction (EF) were calculated.

Continuous variables were expressed as mean \pm \pm standard deviation or median (range) values, and categorical data as percentages. All dichotomous variables were compared applying the $\chi^{2}$ test; continuous variables using one-way analysis of variance. Changes from baseline to follow-up were assessed using analysis of variance for repeated measures. Bonferroni's correction was utilized for multiple comparisons. $\mathrm{P}<0.05$ was considered statistically significant.

Our study population comprised 25 patients hospitalized for acute respiratory failure due to

Address for correspondence: Rosario Rossi, Associate Professor of Cardiology, University of Modena and Reggio Emilia (UNIMORE), Policlinico di Modena, Via del Pozzo, 71, 41124 Modena, Italy, tel: 0039059 4225630, fax: 0039059 4223714, e-mail: rosario.rossi@unimore.it

Received: 16.07.2021 Accepted: 20.11.2021 Early publication date: 1.12 .2021

This article is available in open access under Creative Common Attribution-Non-Commercial-No Derivatives 4.0 International (CC BY-NC-ND 4.0) license, allowing to download articles and share them with others as long as they credit the authors and the publisher, but without permission to change them in any way or use them commercially. 
Table 1. Comparison between symptomatic (study group) and asymptomatic (control group) post-COVID-19 patients. Both groups consisted of patients who survived SARS-CoV-2 infection, but only patients included in the study group suffered from the specific cluster of the following signs and symptoms: asthenia, fatigue, elevated heart rate at rest, and tachycardia disproportionate to exertion. The control group consisted of asymptomatic patients of equal age and gender.

\begin{tabular}{|c|c|c|c|}
\hline Parameter & $\begin{array}{l}\text { Control (asymptomatic) } \\
\text { group }(n=25)\end{array}$ & $\begin{array}{l}\text { Study (symptomatic) } \\
\text { group }(\mathrm{n}=25)\end{array}$ & $\mathbf{P}$ \\
\hline Age [years] & $64 \pm 4$ & $64 \pm 5$ & 0.9 \\
\hline Male gender & $72 \%(n=18)$ & $72 \%(n=18)$ & 1 \\
\hline $\mathrm{BSA}\left[\mathrm{m}^{2}\right]$ & $2.1 \pm 0.5$ & $2.4 \pm 0.6$ & 0.8 \\
\hline BMI $\left[\mathrm{kg} / \mathrm{m}^{2}\right]$ & $26.7 \pm 2.7$ & $27.6 \pm 2.6$ & 0.6 \\
\hline SBP [mmHg] & $125 \pm 9$ & $110 \pm 8$ & 0.01 \\
\hline $\mathrm{DBP}[\mathrm{mmHg}]$ & $74 \pm 7$ & $78 \pm 9$ & 0.01 \\
\hline WHO functional class: & & & 0.0001 \\
\hline 0 & $100 \%(n=25)$ & 0 & \\
\hline I & 0 & 0 & \\
\hline II & 0 & $24 \%(n=6)$ & \\
\hline III & 0 & $76 \%(n=19)$ & \\
\hline IV & 0 & 0 & \\
\hline $6 \mathrm{MWT}[\mathrm{m}]$ & $590 \pm 40$ & $420 \pm 45$ & 0.0001 \\
\hline $\mathrm{HR}$ at rest [bpm] & $73 \pm 14$ & $91 \pm 10$ & 0.001 \\
\hline HR peak, on maximum physical effort [bpm] & $110 \pm 13$ & $135 \pm 17$ & 0.001 \\
\hline \multicolumn{4}{|l|}{ Echocardiographic and Doppler parameters } \\
\hline RV EDD (basal) [mm] & $45 \pm 8$ & $55 \pm 9$ & 0.001 \\
\hline RV:LV ratio & $0.9 \pm 0.3$ & $1.2 \pm 0.3$ & 0.01 \\
\hline RV length [mm] & $62 \pm 13$ & $71 \pm 14$ & 0.03 \\
\hline RVOT diameter [mm] & $30 \pm 7$ & $32 \pm 8$ & 0.3 \\
\hline TAPSE $[\mathrm{mm}]$ & $23 \pm 3$ & $16 \pm 3$ & 0.001 \\
\hline SPAP $[\mathrm{mmHg}]$ & $26 \pm 4$ & $42 \pm 5$ & 0.001 \\
\hline RV EF (3D) [\%] & $50 \pm 5$ & $41 \pm 6$ & 0.001 \\
\hline $\mathrm{RA}$ area $\left[\mathrm{cm}^{2}\right]$ & $13 \pm 4$ & $19 \pm 3$ & 0.001 \\
\hline \multicolumn{4}{|l|}{ Hemodynamic parameters } \\
\hline $\mathrm{mRAP}[\mathrm{mmHg}]$ & $4 \pm 2$ & $11 \pm 3$ & 0.001 \\
\hline $\mathrm{mPAP}[\mathrm{mmHg}]$ & $16 \pm 3$ (range: $13-19)$ & $23 \pm 2$ (range: $21-27)$ & 0.01 \\
\hline PCWP $[\mathrm{mmHg}]$ & $9 \pm 2$ & $8 \pm 2$ & 0.8 \\
\hline mRAP/PCWP & $0.44 \pm 0.22$ & $1.38 \pm 0.29$ & 0.0001 \\
\hline PVR [Wood Unit] & $1.5 \pm 0.6$ & $3.1 \pm 0.7$ & 0.01 \\
\hline $\mathrm{Cl}\left[\mathrm{L} / \mathrm{min} / \mathrm{m}^{2}\right]$ & $2.7 \pm 0.6$ & $2.2 \pm 0.4$ & 0.01 \\
\hline RV SV [mL/beat] & $77 \pm 16$ & $59 \pm 14$ & 0.001 \\
\hline RV EDD [mm] & $48 \pm 13$ & $59 \pm 12$ & 0.001 \\
\hline RV EF [\%] & $55 \pm 9$ & $43 \pm 9$ & 0.001 \\
\hline
\end{tabular}

$\mathrm{BMI}$ — body mass index; BSA — body surface area; $\mathrm{Cl}$ — cardiac index; DBP — diastolic blood pressure; HR — heart rate; LV — left ventricle; $\mathrm{mPAP}$ - mean pulmonary artery pressure; mRAP - mean right atrial pressure; PCWP — pulmonary capillary wedge pressure; PVR — pulmonary vascular resistance; RA — right atrium; RV — right ventricle; RV EDD — right ventricular end-diastolic diameter; RV EF — right ventricular ejection fraction; RVOT — right ventricular outflow tract; RV SV — right ventricular stroke volume; SBP — systolic blood pressure; SPAP — systolic pulmonary artery pressure; WHO - World Health Organization; 6MWT - 6-minute walking test

COVID-19 interstitial pneumonia, confirmed by chest computed tomography images.

The comparison between symptomatic patients and the control group is depicted in Table 1.
Twenty-one patients/25 (84\% of the entire population) had an additional control after 6 months, and $15 / 25(60 \%)$ one more after 1 year of follow-up. In these patients we noted a significant improve- 
ment in pulmonary pressure (mPAP passed from $23 \pm 2$ to $20 \pm 4 \mathrm{mmHg}$ after 6 months, to $18 \pm$ $\pm 5 \mathrm{mmHg}$ after 1 year of follow-up; $\mathrm{p}=0.01$ for all comparisons); a significant increase in RV systolic performance (RVSV moved from $59 \pm 8$ to $66 \pm$ $\pm 10 \mathrm{~mL}$ at 6 months, and $72 \pm 12 \mathrm{~mL}$ after 1 year; $\mathrm{p}=0.01$ ); associated with a significant amelioration of 6MWT (from $420 \pm 45$ to $495 \pm 40 \mathrm{~m}$ after 6 months, to $520 \pm 35 \mathrm{~m}$ after 1 year; $\mathrm{p}=0.001$ ).

All patients who presented the following cluster of signs/symptoms: asthenia, fatigue, high HR at rest, and tachycardia disproportionate to physical exertion, suffered from mild degree pulmonary arterial hypertension $(\mathrm{PAH})$ and severe RV systolic dysfunction. We found a perfect match between this hemodynamic status and the described clinical condition.

All our symptomatic patients presented a mPAP $>20 \mathrm{mmHg}$, a value already indicative of $\mathrm{PH}$ according to the $6^{\text {th }}$ World Symposium on Pulmonary Hypertension of Nice, 2018 [4]. In addition, RVSV and EF were both reduced by about $40 \%$ compared to the asymptomatic (control) group.

Our hypothesis would be that, in relation to the parenchymal ("ground-glass" and "dense" opacifications) and vascular pulmonary involvement (markedly impaired pulmonary perfusion, caused by pulmonary angiopathy and thrombosis) that occurs during the acute phase of pneumonia $[5,6]$, an increase in PVR develops, with a consequent onset of PH [7]. RV normally works at an extremely low level of afterload, and the muscle thickness of its free wall is not particularly conspicuous. However, when the RV is stressed by an increase in afterload, its internal cavity gradually expands [8, 9]. In this phase of the disease, the right atrium acts as an effective pump in the late diastole. The pressure in the right atrium consequently increases. The intensification of the right atrium contractile efficiency implies a recruitment of preload, which has the function of more effectively filling the underlying ventricle. According to the Frank-Starling principle, the RV increases the stroke volume [9].

Furthermore, the most important and timely compensatory mechanism capable of maintaining an adequate $\mathrm{CO}$, represented by the stimulation of the sympathetic-adrenaline reflex, becomes active from the earliest stages of the disease, which leads to positive chronotropic and inotropic effects [10].

In conclusion, there is a phase of COVID-19, after discharge, characterized by $\mathrm{PAH}$ and RV systolic dysfunction. As long as this state persists, patients suffer from a specific cluster of symptoms and signs.

Our results allow us to assume that recovery of normal RV function is gradual and spontaneous. In other words, the RV initially sustains an increase in pulmonary resistance, and then gradually resumes its function, as after a "stunning". However, it is noticeable that the disappearance of symptoms is always accompanied by improvement of the RV systolic performance.

\section{Conflict of interest: None declared}

\section{References}

1. Belli S, Balbi B, Prince I, et al. Low physical functioning and impaired performance of activities of daily life in COVID-19 patients who survived hospitalisation. Eur Respir J. 2020; 56(4), doi: 10.1183/13993003.02096-2020, indexed in Pubmed: 32764112.

2. Sigfrid L, Cevik M, Jesudason E, et al. What is the recovery rate and risk of long-term consequences following a diagnosis of COVID-19? A harmonised, global longitudinal observational study protocol. BMJ Open. 2021; 11(3): e043887, doi: 10.1136/ bmjopen-2020-043887, indexed in Pubmed: 33692181.

3. Moreno-Pérez O, Merino E, Leon-Ramirez JM, et al. COVID-19-ALC research group. Post-acute COVID-19 syndrome. Incidence and risk factors: A Mediterranean cohort study. J Infect. 2021; 82(3): 378-383, doi: 10.1016/j.jinf.2021.01.004, indexed in Pubmed: 33450302 .

4. Galiè N, McLaughlin VV, Rubin LJ, et al. An overview of the 6th World Symposium on Pulmonary Hypertension. Eur Respir J. 2019; 53(1), doi: 10.1183/13993003.02148-2018, indexed in Pubmed: 30552088.

5. Patel BV, Arachchillage DJ, Ridge CA, et al. Pulmonary angiopathy in severe COVID-19: physiologic, imaging, and hematologic observations. Am J Respir Crit Care Med. 2020; 202(5): 690-699, doi: 10.1164/rccm.202004-1412OC, indexed in Pubmed: 32667207.

6. Jha AK. Pulmonary vascular changes in acute respiratory distress syndrome due to COVID-19. Am J Respir Crit Care Med. 2021; 203(2): 259-260, doi: 10.1164/rccm.202009-3508LE, indexed in Pubmed: 33085907.

7. Chen L, Tang R, Chen H, et al. Pulmonary vasculature: a target for COVID-19. Am J Respir Crit Care Med. 2021; 203(2): 260-261, doi: 10.1164/rccm.202009-3564LE, indexed in Pubmed: 33085902 .

8. Amsallem M, Boulate D, Aymami M, et al. Load adaptability in patients with pulmonary arterial hypertension. Am J Cardiol. 2017; 120(5): 874-882, doi: 10.1016/j.amjcard.2017.05.053, indexed in Pubmed: 28705377.

9. Vonk-Noordegraaf A, Haddad F, Chin KM, et al. Right heart adaptation to pulmonary arterial hypertension: physiology and pathobiology. J Am Coll Cardiol. 2013; 62 (25 Suppl): D22-D33, doi: 10.1016/j.jacc.2013.10.027, indexed in Pubmed: 24355638.

10. El Hajj MC, Viray MC, Tedford RJ. Right heart failure: a hemodynamic review. Cardiol Clin. 2020; 38(2): 161-173, doi: 10.1016/j. ccl.2020.01.001, indexed in Pubmed: 32284094. 